\title{
Onfalocele: Prognóstico Fetal em 51 Casos com Diagnóstico Pré-natal
}

\author{
Omphalocele: Fetal Prognosis in 51 Cases with Prenatal Diagnosis
}

Samir Aldalla Mustafá ${ }^{1}$, Maria de Lourdes Brizot ${ }^{1}$, Mário Henrique Burlacchinite Carvalho ${ }^{1}$, Maria Okumura ${ }^{1}$, Lilian Patroni Toro ${ }^{1}$, Marcos Marques Silva², Marcelo Zugaib ${ }^{1}$

\section{RESUMO}

Objetivo: avaliar o prognóstico fetal dos casos de onfalocele com diagnóstico pré-natal.

Métodos: foram analisados 51 casos de onfalocele com diagnóstico pré-natal e divididos em 3 grupos: Grupo 1, onfalocele isolada; Grupo 2, onfalocele com malformações estruturais associadas e cariótipo normal; Grupo 3, onfalocele associada à cromossomopatia. As análises foram realizadas em relação à sobrevida geral e pós-correção cirúrgica, considerando as malformações associadas, idade gestacional no parto, peso no nascimento e tamanho da onfalocele.

Resultados: o Grupo 1 correspondeu a 21\% (n=11), o Grupo 2 a 55\% $(n=28)$ e o Grupo 3 a 24\% ( $n=12)$. Todos os casos do grupo 3 evoluiram para óbito, e a cromossomopatia mais freqüente foi a trissomia do 18. A sobrevida foi de 80\% no Grupo 1 e de 25\% no Grupo 2. Dezesseis casos foram submetidos à correção cirúrgica (10 isoladas e 6 associadas) e 81\% sobreviveram (8 isoladas e 5 associadas). A mediana do peso no nascimento dos sobreviventes pós-correção cirúrgica foi $3.140 \mathrm{~g}$ e dos que morreram foi de $2.000 \mathrm{~g}(\mathrm{p}=0,148)$ e a idade gestacional do parto foi de 37 e de 36 semanas $(p=0,836)$, respectivamente. A relação das circunferências onfalocele/abdominal diminuiu com a idade gestacional, 0,88 entre 25-29 semanas e 0,65 entre 30-35 semanas ( $p=0,043)$. Não foi observada diferença significativa no tamanho da onfalocele nos 3 grupos $(p=0,988)$ e influência deste prognóstico pós-correção cirúrgica ( $p=0,553)$.

Conclusão: a sobrevida geral e pós-correção cirúrgica foi de 25 e $81 \%$, respectivamente. As malformações associadas representam o principal fator prognóstico das onfaloceles com diagnóstico pré-natal, visto que se associam com prematuridade e baixo peso.

PALAVRAS-CHAVE: Diagnóstico pré-natal. Malformação fetal. Mortalidade perinatal. Onfalocele.

\section{Introdução}

Onfalocele é um defeito na parede abominal, na inserção do cordão umbilical, com herniação de órgãos abdominais ${ }^{1}$. Apresenta uma incidência de 2,5 em 10.000 nascidos vi$\operatorname{vos}^{2}$. O diagnóstico pré-natal baseia-se na demons-

\footnotetext{
${ }^{1}$ Setor de Medicina Fetal da Clínica Obstétrica do Hospital das Clínicas da FMUSP

${ }^{2}$ Disciplina de Cirurgia Pediátrica do Departamento de Pediatria do Instituto da Criança do HCFMUSP.

Correspondência:

Maria de Lourdes Brizot

Clinica Obstétrica - ICHC/FMUSP

Av. Dr. Enéas de Carvalho Aguiar, 225 10o andar

05403-900 - São Paulo - SP

e-mail:mlbrizot@uol.com.br
}

tração do defeito na linha mediana da parede abdominal, com presença de saco herniário com conteúdo visceral no qual se insere o cordão umbilical. O defeito é caracterizado pela ausência dos músculos abdominais, fáscia e pele e, coberto por uma membrana avascular, forma uma hérnia. $\mathrm{O}$ saco herniado é formado por uma camada interna (peritônio) e uma externa (membrana amniótica) e entre as duas existe uma fina camada de geléia de Wharton. A migração das alças intestinais no cordão umbilical ocorre normalmente entre $8 \mathrm{e}$ 12 semanas de gestação ${ }^{3}$ e falha no retorno das alças intestinais para a cavidade abdominal resulta na formação de onfalocele.

A embriogênese da onfalocele não é muito bem entendida. Várias teorias foram sugeridas 
para esclarecer sua origem. Em casos de onfaloceles extensas, nas quais há herniação de figado e alças intestinais, o defeito pode resultar da interrupção no desenvolvimento dos folhetos laterais e de falha no fechamento da parede, por volta da terceira ou quarta semana de vida embrionária. Porém, nas onfaloceles pequenas, em que somente as alças intestinais estão herniadas, o defeito pode ser resultante de uma falha nos estágios finais de fechamento dos folhetos laterais, secundário à exposição a agentes teratogênicos ou alterações genéticas que predispõem ao desenvolvimento de malformações ${ }^{4}$. Todos os órgãos abdominais podem herniar, sendo mais freqüente a protusão de alças intestinais, estômago e figado.

A associação de onfalocele com anomalias cromossômicas ocorre em 8 a $67 \%$ dos $\operatorname{casos}^{5-7}$, sendo freqüente as trissomias do 18,13 e 21 . A incidência de anomalias cromossômicas é maior na presença de outras malformações e quando a onfalocele é pequena, contendo somente o omento ou intestino ${ }^{4}$.

Algumas sindromes genéticas também podem se apresentar com onfalocele, tais como a pentalogia de Cantrel (onfalocele, defeitos do esterno, do diafragma anterior e defeitos cardiacos) e a sindrome de Beckwith-Wiedemann (onfalocele, macroglossia e gigantismo) ${ }^{8}$.

Fetos com onfalocele apresentam alta mortalidade, que varia de acordo com a presença de malformações ou cromossomopatias associadas. Quando isolada, o prognóstico é muito bom, com uma taxa de sobrevida de até $94 \%{ }^{9}$.

Este estudo tem por objetivo avaliar o prognóstico das onfaloceles com diagnóstico pré-natal.

\section{Pacientes e Métodos}

Foi realizado levantamento retrospectivo dos casos de onfalocele com diagnóstico pré-natal, atendidos no Setor de Medicina Fetal do Hospital das Clínicas da FMUSP, no período de janeiro de 1992 a abril de 2000.

Para o diagnóstico de onfalocele consideramos a presença de protrusão dos órgãos abdominais envolvidos por uma membrana e com inserção do cordão umbilical no saco herniário.

As medidas da onfalocele foram realizadas em corte transversal na abertura da parede abdominal. Foi considerada onfalocele grande aquela com diâmetro do saco herniário maior que 5 $\mathrm{cm}$. Também foi calculada a razão entre a circunferência da onfalocele e a circunferência abdominal.
O conteúdo herniado foi discriminado de acordo com os órgãos exteriorizados em: intestino; figado; intestino e figado; figado e estômago; figado e coração; figado, intestino e estômago; fígado, intestino e coração. Os fetos com defeito extenso da parede abdominal anterior foram excluídos.

A avaliação morfológica detalhada e a ecocardiografia fetal foram realizadas em todos os casos para diagnóstico de outras malformações associadas. Após a confirmação do diagnóstico, foi realizado aconselhamento genético e oferecido análise do cariótipo fetal por meio de procedimentos invasivos como a amniocentese ou a cordocentese.

As informações sobre os fetos que nasceram neste serviço foram obtidas dos prontuários, e sobre os nascidos em outros hospitais, por contacto telefônico ou carta-resposta.

Para análise dos resultados, foram considerados: idade materna, idade gestacional no diagnóstico, cromossomopatias associadas, malformações associadas, tamanho da onfalocele, conteúdo da onfalocele, volume de líquido amniótico, idade gestacional no parto, peso no nascimento, sexo do recém-nascido, evolução da gestação e evolução pós-correção cirúrgica.

Para melhor avaliar a evolução e prognóstico da gestação, dividimos os casos em 3 grupos: Grupo 1, onfalocele isolada sem anomalias cromossômicas (Tabela 1); Grupo 2, onfalocele associada a outras malformações sem anomalias cromossômicas (Tabela 2); Grupo 3, onfalocele com anomalia cromossômica (isolada ou associada; Tabela 3).

Tabela 1 - Evolução das onfaloceles isoladas com cariótipo normal (grupo 1).

ID = idade materna; IGD = idade gestacional no diagnóstico; IGP = idade gestacional ao parto; $\mathrm{P}=$ tipo de parto; $\mathrm{ACH}=$ achados; $\mathrm{EG}=$ evolução da gestação; $C O N T$ = conteúdo da onfalocele; TAM = tamanho da onfalocele.

\begin{tabular}{cccccccccc}
\hline CASO & ID & IGD & SEXO & CONT & TAM & IGP & P & PESO & EG \\
\hline 1 & 28 & 30 & $\mathrm{M}$ & $\mathrm{F}$ & $>5 \mathrm{~cm}$ & 35 & $\mathrm{C}$ & 1800 & Onn \\
2 & 34 & 32 & $\mathrm{M}$ & $\mathrm{I}$ & $<5 \mathrm{~cm}$ & 39 & $\mathrm{C}$ & 3295 & vivo \\
3 & 30 & 33 & $\mathrm{NM}$ & $\mathrm{I}$ & $\mathrm{NM}$ & $\mathrm{NM}$ & $\mathrm{NM}$ & $\mathrm{NM}$ & vivo \\
4 & 23 & 27 & $\mathrm{~F}$ & $\mathrm{~F}+1+\mathrm{E}$ & $>5 \mathrm{~cm}$ & 39 & $\mathrm{C}$ & 4180 & vivo \\
5 & 20 & 35 & $\mathrm{M}$ & $\mathrm{I}$ & $>5 \mathrm{~cm}$ & 39 & $\mathrm{~V}$ & 3780 & vivo \\
6 & 25 & 33 & $\mathrm{~F}$ & $\mathrm{~F}$ & $>5 \mathrm{~cm}$ & 37 & $\mathrm{C}$ & 2590 & vivo \\
7 & 29 & 27 & $\mathrm{~F}$ & $\mathrm{~F}$ & $>5 \mathrm{~cm}$ & 38 & $\mathrm{C}$ & 3520 & vivo \\
8 & 23 & 28 & $\mathrm{M}$ & $\mathrm{F}$ & $>5 \mathrm{~cm}$ & 30 & $\mathrm{NM}$ & $\mathrm{NM}$ & RPM-onn \\
9 & 19 & 32 & $\mathrm{~F}$ & $\mathrm{~F}+\mathrm{I}$ & $<5 \mathrm{~cm}$ & 36 & $\mathrm{C}$ & 2400 & vivo \\
10 & 22 & 26 & $\mathrm{M}$ & $\mathrm{F}$ & $>5 \mathrm{~cm}$ & 36 & $\mathrm{C}$ & 2270 & vivo \\
\hline
\end{tabular}

$\mathrm{M}=$ masculino; $\mathrm{F}=$ feminino; $\mathrm{NM}=$ não mencionado; $\mathrm{v}$ = vaginal; $\mathrm{c}=$ cesárea; onf = onfalocele; onn = óbito neonatal; RPM = rotura prematura das membranas; $\mathrm{F}$ = fígado; I = intestino; $\mathrm{E}$ = estômago. 
Tabela 2 - Evolução das onfaloceles associadas a outras malformações com cariótipo normal (grupo 2). IM = Idade materna; IGD = idade gestacional no diagnóstico; MFs ASSOC = malformações associadas; IGP = idade gestacional ao parto; TP = tipo de parto; EG = evolução da gestação; CONT = conteúdo da onfalocele; TAM = tamanho da onfalocele.

\begin{tabular}{|c|c|c|c|c|c|c|c|c|c|c|}
\hline CASO & IM & IGD & MFs ASSOC & Sexo & CONT & TAM & IGP & TP & PESO & EG \\
\hline 1 & 22 & 28 & Hidronefrose+ ventriculomegalia & $\mathrm{F}$ & $F+I$ & NM & 32 & c & 1800 & vivo \\
\hline 2 & 30 & 28 & Cardio (CIV) & $\mathrm{F}$ & $\mathrm{F}$ & $<5 \mathrm{~cm}$ & 39 & $c$ & 3200 & Vivo \\
\hline 3 & 27 & 27 & Hérnia diafragmática+ Auu & $\mathrm{F}$ & $\mathrm{F}$ & $<5 \mathrm{~cm}$ & 40 & c & 2650 & Onn - não foi p/ cirurgia \\
\hline 4 & 29 & 26 & Ventriculomegalia & $\mathrm{F}$ & $\mathrm{F}$ & NM & 33 & c & 3280 & Onn \\
\hline 5 & 18 & 32 & Coluna torta+pé torto bilateral & $\mathrm{F}$ & $\mathrm{F}$ & NM & $?$ & v & $?$ & Onn \\
\hline 6 & 32 & 13 & Coluna torta+pé torto bilateral & $\mathrm{F}$ & $F+I+E$ & $<5 \mathrm{~cm}$ & 36 & v & 1760 & Onn \\
\hline 7 & 31 & 28 & Spina bífida+CIV+pé torto bilateral & $\mathrm{F}$ & $F+1$ & $<5 \mathrm{~cm}$ & 31 & $\mathrm{v}$ & 2500 & Onn - I J \\
\hline 8 & 28 & 35 & $\mathrm{H}$ de VE+rins policísticos+Auu & $\mathrm{F}$ & $\mathrm{F}$ & $<5 \mathrm{~cm}$ & $?$ & $\mathrm{v}$ & $?$ & Oiu \\
\hline 9 & 22 & 26 & $\begin{array}{l}\text { Cisto porencefálico+pé torto } \\
\text { bilateral+rins policísticos }\end{array}$ & $\mathrm{F}$ & NM & NM & 29 & $\mathrm{c}$ & 1380 & Oiu \\
\hline 10 & 22 & 27 & $\begin{array}{l}\text { Ventriculomegalia+rins policísticos+pé } \\
\text { torto bilateral }\end{array}$ & $\mathrm{F}$ & $\mathrm{F}$ & NM & 28 & v & 980 & Oiu \\
\hline 11 & 24 & 33 & $\begin{array}{l}\text { Exencefalia+retrognatia+mãos em } \\
\text { garra+pé torto bilateral+ Auu }\end{array}$ & $\mathrm{F}$ & $F+E$ & NM & 33 & $v$ & 1340 & Oiu \\
\hline 12 & 29 & 36 & Hidrocefalia+lábio leporino & $\mathrm{F}$ & $F+I+E$ & NM & 36 & v & 3000 & Onn - não foi p/ cirurgia \\
\hline 13 & 23 & 26 & Hidrocefalia & M & I & $<5 \mathrm{~cm}$ & 29 & v & 1010 & Oiu \\
\hline 14 & 21 & 31 & Macroglossia+ visceromegalia & M & I & $>5 \mathrm{~cm}$ & 37 & c & 4950 & sd Beckwith Wiedman - Vivo \\
\hline 15 & 32 & 33 & Ascite & M & $F+E$ & $>5 \mathrm{~cm}$ & 35 & c & $?$ & Vivo \\
\hline 16 & 24 & 32 & Coluna torta+pé torto bilateral & M & $F+E$ & NM & 38 & $v$ & 1870 & Onn \\
\hline 17 & 32 & 20 & Coluna torta+Auu & M & NM & N M & 21 & c & 380 & Oiu \\
\hline 18 & 19 & 32 & Auu & M & $\mathrm{F}$ & $<5 \mathrm{~cm}$ & 34 & v & 1880 & Vivo \\
\hline 19 & 40 & 27 & Cardio (CIV) & M & $F+E$ & $>5 \mathrm{~cm}$ & 36 & c & 3080 & Op 1 ddv ob 3 meses \\
\hline 20 & 28 & 32 & Hidranencefalia+pé torto bilateral & M & $F+1$ & NM & 36 & v & 1570 & Oiu \\
\hline
\end{tabular}

Cardio = Malformação cardíaca; $\mathrm{CIV}=$ comunicação inter-ventricular; $\mathrm{Auu}=$ artéria umbilical única; $\mathrm{H}$ de VE = hipoplasia de ventriculo esquerdo; $\mathrm{M}=$ masculino; $\mathrm{F}=$ feminino; $\mathrm{V}=$ vaginal c = cesárea; Onn = óbito neonatal; Oiu = óbito intra-útero; IJ = interrupção judicial; sd = síndrome; Op = operado; ddv = dia de vida; ob = óbito; $F=$ fígado; I = intestino; $E$ = estômago; $N M$ = não mencionado.

Tabela 3 - Evolução das onfaloceles com cariótipo anormal (grupo 3). IM = Idade materna; IGD = idade gestacional no diagnóstico; CARIO= cariótipo; IGP = idade gestacional no parto; $\mathrm{TP}=$ tipo de parto; $\mathrm{EG}=$ evolução da gestação; CONT = conteúdo da onfalocele; TAM = tamanho da onfalocele.

\begin{tabular}{|c|c|c|c|c|c|c|c|c|c|}
\hline CASO & IM & IGD & CARIO & CONT & TAM & IGP & TP & PESO & EG \\
\hline 1 & 32 & 21 & $m(x x / 18)$ & I & $?$ & & v & 1000 & Oiu \\
\hline 2 & 40 & 23 & $47 x y+18$ & $\mathrm{~F}, \mathrm{I}$ & $?$ & 26 & v & $?$ & Oiu - IJ \\
\hline 3 & 41 & 34 & $47 x y+18$ & $\mathrm{~F}, \mathrm{I}$ & $<5 \mathrm{~cm}$ & & $\mathrm{v}$ & 1100 & Oiu \\
\hline 4 & 43 & 28 & $47 x y+18$ & $\mathrm{~F}$ & $?$ & 34 & $\mathrm{v}$ & 910 & Onn \\
\hline 5 & 19 & 30 & $47 x y+18$ & $\mathrm{~F}$ & $>5 \mathrm{~cm}$ & 35 & $\mathrm{v}$ & 1050 & Onn \\
\hline 6 & 27 & 30 & $47 x y+18$ & $\mathrm{~F}, \mathrm{I}$ & $?$ & 35 & v & 1600 & Oiu - IJ \\
\hline 7 & 37 & 36 & $47 x x+18$ & $\mathrm{~F}$ & $<5 \mathrm{~cm}$ & 37 & $\mathrm{v}$ & $?$ & Onn \\
\hline 8 & 26 & 26 & $47 x x+18$ & $\mathrm{~F}, \mathrm{I}$ & $<5 \mathrm{~cm}$ & 30 & $\mathrm{v}$ & $?$ & Onn \\
\hline 9 & 35 & 27 & $47 x x+18$ & I & $?$ & & $\mathrm{v}$ & $?$ & Onn \\
\hline 10 & 36 & 26 & $47 x x+18$ & $\mathrm{~F}, \mathrm{I}$ & $>5 \mathrm{~cm}$ & & $\mathrm{v}$ & 2000 & Oiu \\
\hline 11 & 22 & 30 & $46 x y \operatorname{inv} 9$ & $\mathrm{~F}$ & $>5 \mathrm{~cm}$ & 38 & $c$ & 750 & Op $3 d d v$, ob ddv \\
\hline 12 & 18 & 23 & $46 x x+13$ & $\mathrm{~F}, \mathrm{I}$ & $>5 \mathrm{~cm}$ & 32 & v & & Oiu \\
\hline
\end{tabular}

v = Vaginal; c = cesáreo; Oiu = óbito intra-útero; Onn = óbito neonatal; IJ = interrupção judicial; Op = operado; ddv = dia de vida; F= fígado; I = intestino; ob = óbito.

$\mathrm{Na}$ análise estatística foi utilizado o teste de Mann-Whitney para comparação da idade ges- tacional no parto e peso no nascimento entre os grupos. Na avaliação da evolução da relação do ta- 
manho das circunferências onfalocele/abdome com a idade gestacional foi utilizado o teste de Wilcoxon. Na comparação da relação onfalocele/ abdome nos grupos 1, 2 e 3 foi utilizado o teste de Kruskal-Wallis. Os testes foram considerados significativos quando $\mathrm{p}<0.05$.

\section{Resultados}

Foram analisados 51 casos de onfalocele. A onfalocele isolada foi observada em 11 casos $(22 \%)$. Em 40 casos foram detectadas outras anomalias estruturais (78\%). Neste grupo, 7 não foram submetidas à análise do cariótipo fetal e em outros 2 houve falha de cultura.

Detalhes específicos de cada caso, nos diferentes grupos, estão discriminados nas Tabelas 1,2 e 3.

A idade materna média foi de 27 anos (faixa de variação de 18-43 anos) no grupo das onfaloceles isoladas, de 28 anos (de 18-43) nas associadas às outras anomalias estruturais e de 26 anos (de 18-43) nas onfaloceles com anomalias cromossômicas. A idade gestacional média no diagnóstico foi de 28 semanas (faixa de variação de 13-36).

As malformações associadas mais observadas em ordem de freqüência foram: esqueléticas $(39 \%)$, cardiacas $(29 \%)$, sistema nervoso central $(28 \%)$, coluna $(25 \%)$ e trato gênito-urinário $(10 \%)$.

Procedimento invasivo para análise de cariótipo fetal (cordocentese ou amniocentese) foi realizado em 43 casos (84\%) e o resultado do cariótipo obtido em 41 casos. O cariótipo foi normal em $71 \%$ e anormal em $29 \%$. A trissomia do 18 foi a anomalia cromossômica mais freqüente. Em 83\% (10/12) das onfaloceles com cromossomopatias havia outras malformações associadas.

O líquido amniótico (LA) estava alterado em $40 \%$ das gestações $(29 \%$ com poliidrâmnio e $11 \%$ com oligoidrâmnio). Observamos uma maior associação de poliidrâmnio nos grupos 2 e 3, 45\% $(n=9 / 20)$ e $41 \%(n=5 / 12)$, respectivamente.

A mediana da idade gestacional no parto, no grupo geral, foi de 35 semanas (faixa de variação de 21-40 semanas), sendo: 37 semanas (3039) no Grupo 1, 33 semanas (21-40) no Grupo 2 e 34.5 semanas (26-38) no Grupo 3. Observouse diferença significativa na idade gestacional do parto entre os Grupos 1 e $2(p=0,01)$. Entretanto, na comparação entre os Grupos 1 e 3 a diferença não foi estatisticamente significativa, mas parece haver uma tendência para uma idade gestacional menor no grupo com anomalia cromossômica $(p=0,05)$. A mediana da idade gestacional do parto do grupo que sobreviveu após correção cirúrgica foi de 37 semanas e do grupo que morreu foi de 36 semanas.

A taxa de prematuridade (idade gestacional do parto $<37$ semanas) no grupo geral foi de $73 \%(30 / 41)$. Não foi possivel obter idade gestacional no parto em 10 casos. A prematuridade esteve presente em 44\% (4/9) no Grupo 1, ocorrendo óbito neonatal em dois dos casos. No Grupo 2 a taxa de manutenção foi de $77 \%$ (14/18), ocorrendo 6 casos de óbito intra-útero e 4 de óbito neonatal, e de $75 \%$ (6/8) no Grupo 3. Entre os nascidos vivos a taxa de prematuridade foi de $62 \%$ e nos que sobreviveram pós-correção cirúrgica foi de $46 \%(6 / 13)$, comparada com $63.3 \%$ $(2 / 3)$ nos que morreram pós-correção cirúrgica.

Em 27 casos o parto foi vaginal (Grupo 1, $\mathrm{n}=1$; Grupo 2, $\mathrm{n}=15$; Grupo $3, \mathrm{n}=11$ ). Parto cesáreo foi realizado em 19 casos (Grupo 1, n = 8; Grupo 2, n = 10; Grupo 3, n = 1). Em 5 casos não foi obtido resultado da gestação.

No grupo geral, a mediana do peso médio no nascimento foi de $1.800 \mathrm{~g}$ (faixa de variação de 350-4.950), sendo que nos diferentes grupos assim se mostrou: Grupo $1=2.934 \mathrm{~g}$ (1.800$4.180 \mathrm{~g})$; Grupo $2=1.780 \mathrm{~g}$ (350-4.950); Grupo $3=1.050 \mathrm{~g}$ (750-2.000). Na comparação do peso entre os grupos foi observada diferença estatisticamente significativa entre o peso dos Grupos 1 e $2(\mathrm{p}=0,01)$ e os Grupos 1 e 3 (p=0,002). Comparando-se o peso no nascimento dos que sobreviveram com os que morreram, pós correção cirúrgica, não houve diferença estatisticamente significativa $(p=0,14)$.

Dezesseis recém-nascidos foram submetidos à correção cirúrgica, dos quais 13 sobreviveram no período neonatal $(81 \%)$. Dos sobreviventes pós-cirurgia, cinco dos treze apresentavam outras anomalias estruturais associadas: hidronefrose leve e ventriculomegalia $(\mathrm{n}=1)$; sindrome de Beckwith-Wiedemann ( $\mathrm{n}=1)$, comunicação interventricular $(\mathrm{n}=1)$, ascite $(\mathrm{n}=1)$ e artéria umbilical única $(\mathrm{n}=1)$. Três casos evoluíram para óbito pós-correção cirúrgica: dois no periodo neonatal (um caso de onfalocele isolada evoluiu para óbito por insuficiência respiratória e o outro apresentava cardiopatia associada (dupla via de saída de ventriculo direito) e um com 3 meses de vida, com cardiopatia associada (CIV = comunicação interventricular), devido à infecção.

Em relação ao conteúdo herniário, 34\% (n $=17 / 51)$ apresentava apenas figado; $21 \%(\mathrm{n}=$ $11 / 51$ ) apenas alças intestinais; em $21 \%$ havia herniação de alças intestinais e figado; em $10 \%$ ( $\mathrm{n}=5 / 51)$, figado, alças intestinais e estômago 
herniados; em $8 \%(n=4 / 51)$, figado e estômago; figado e coração herniados foram observados em $2 \%$. Em 2 casos o conteúdo herniado não foi descrito.

Em 29 casos foi possivel analisar o tamanho da onfalocele em relação ao resultado do cariótipo fetal. Dos sete casos que apresentaram cromossomopatias, em 3 a onfalocele foi menor do que $5 \mathrm{~cm}$ e em 4 maior do que $5 \mathrm{~cm}$.

Em 13 casos submetidos à correção cirúrgica foi avaliado o tamanho da onfalocele no período pré-natal. Em 9 casos o tamanho foi $\geq 5 \mathrm{~cm}$, sendo que 7 sobreviveram e 2 evoluíram para óbito. Os quatro casos com tamanho $<5 \mathrm{~cm}$ sobreviveram.

A relação circunferência da onfalocele/circunferência abdominal foi de 0,8 no periodo entre 25 e 29 semanas, 0,6 entre 30 e 35 semanas e 0,6 acima de 35 semanas. Em 9 casos as relações foram avaliadas longitudinalmente e foi observada uma redução significativa $(\mathrm{p}=0,043)$ entre a primeira (25-29 semanas) e a segunda medida (3035 semanas), com mediana de 0,88 e 0,65, respectivamente. Não foi observada diferença significativa da relação entre os Grupos 1,2 e $3(\mathrm{p}=$ 0,988 ), assim como entre os que sobreviveram e os que morreram pós correção cirúrgica $(p=0,553)$.

\section{Discussão}

No presente estudo a taxa de outras anomalias associadas à onfalocele foi de $74 \%$, sendo compativel com o observado em outras séries, que varia de 39 a $82 \%^{2,5,10,11}$. Essa variação, provavelmente, se deve ao fato de alguns estudos incluírem apenas os casos com diagnóstico pós-natal e também à diferença na idade gestacional na ocasião do diagnóstico. Sabe-se que muitos fetos com onfalocele evoluem com óbito intra-uterino ou neonatal, antes de serem submetidos à correção cirúrgica. Calzolari et al. ${ }^{2}$, em estudo europeu, relatam 54\% de associação com outras malformações. Neste estudo foram incluídos casos com diagnóstico pré-natal, achados de anatomopatológico e produtos de abortamento; entretanto, a conduta não era padronizada entre os diferentes centros. Com isso, a análise desta associação ficou restrita aos centros que ofereciam o diagnóstico pré-natal e àqueles que registravam as interrupções da gestação.

Observamos associação com anomalias cromossômicas em 29\% dos casos, e na literatura esta associação é observada em 9 a $67 \% \%^{5-7,12}$. As diferenças na incidência de aneuploidia podem estar relacionadas a vários fatores, tais como: idade materna da população estudada, idade gestacional no diagnóstico da malformação, presença de outras malformações associadas, conteúdo e tamanho da onfalocele. Observamos que quanto mais precoce a idade gestacional no diagnóstico mais comum é a associação com cromossomopatias $^{7,12}$. Fetos com anomalias cromossômicas apresentam maior chance de abortamento espontâneo, portanto, quanto maior a idade gestacional no diagnóstico menor a possibilidade de uma cromossomopatia associada. Van Zalen-Sprock et al. ${ }^{12}$ observaram $47 \%$ de aneuploidia em 17 casos com diagnóstico pré-natal precoce (entre 11 e 16 semanas), estando todos estes associados a translucência nucal aumentada ou higroma cístico.

Neste estudo, a anomalia cromossômica mais freqüente foi a trissomia do cromossomo 18 , que é a aneuploidia mais associada a este defeito, seguida pela trissomia do cromossomo 13. Em $17 \%$ dos casos com anomalias cromossômicas não foram observadas outras malformações associadas à onfalocele. Portanto, é importante investigar o cariótipo fetal mesmo nos casos com onfalocele isoladas. Nos dois casos com onfalocele isolada que apresentaram aneuploidias a idade materna era menor do que 35 anos.

Quanto menor o tamanho da abertura na parede abdominal maior a associação com anomalias cromossômicas ${ }^{6}$. Onfalocele contendo somente alças intestinais apresentam associação mais freqüente com anomalias cromossômicas, e estas normalmente tendem a ser de tamanho menor do que as que contêm fígado ${ }^{6,13}$. Nesta série observamos maior associação com cromossomopatias nos casos com fígado e intestino herniados, seguidos pelos casos com somente figado herniado. Não observamos correlação entre o tamanho da onfalocele (medida isolada ou onfalocele/abdome) com a incidência de aneuploidias. Isto poderia ser explicado pela idade gestacional tardia na ocasião do diagnóstico, quando já muitas das aneuploidias foram abortadas espontaneamente.

A influência do tamanho da onfalocele e do conteúdo herniado no prognóstico neonatal é controversa na literatura. Quando o baço e o coração estão herniados a mortalidade é de $100 \%{ }^{14}$. Mayer et al. ${ }^{9}$ observaram que onfaloceles grandes $(>5 \mathrm{~cm})$ estão associadas com mortalidade neonatal mais alta do que as de dimensões pequenas. Dunn e Fonkalsrud ${ }^{15}$ referem maior morbimortalidade em crianças que apresentavam defeitos extensos no abdome devido a um maior grau de desproporção víscero-abdominal, causando um aumento da pressão intra-abdominal, necrose das alças intestinais, obstrução 
venosa do figado e falência renal. Kamata et al. ${ }^{16}$ atribuem a mortalidade à hipoplasia pulmonar presente nos casos de aberturas extensas da parede abdominal. Por outro lado, estudos que avaliaram a relação entre o diâmetro transverso da onfalocele com o diâmetro transverso do abdome não observaram esta diferença na ausência de anomalias associadas ${ }^{13}$. Mabogunje e Mahour ${ }^{17}$ também não encontraram associação entre o tamanho da onfalocele e a mortalidade neonatal (> ou $<$ do que $4 \mathrm{~cm}$ ). No presente estudo o tamanho da onfalocele também não influenciou o prognóstico pós-correção cirúrgica.

Fetos com onfalocele apresentam alta mortalidade, variando de acordo com a presença de malformações ou cromossomopatias associadas. Quando isolada, o prognóstico é muito bom, apresentando uma taxa de sobrevida de até $94 \%{ }^{9}$. Observamos taxa de sobrevida maior no grupo com onfalocele isolada (80\%) comparada com o grupo com anomalias estruturais associadas (25\%). As anomalias associadas mais influentes na mortalidade pós-cirúrgica foram as cardiopatias. No grupo com malformações estruturais sem anomalias cromossômicas a sobrevida foi de apenas $31 \%$.

A sobrevida dos recém-nascidos que foram submetidos à correção cirúrgica foi de $81 \%$ (13/ 16), sendo 8 isoladas e 5 associadas. Dos três casos que evoluíram para óbito pós-correção cirúrgica dois apresentavam onfalocele isolada.

Van Zalen-Sprock et al. ${ }^{12}$ observaram translucência nucal aumentada ou higroma cístico em $47 \%$ ( $\mathrm{n}=8 / 17)$ dos casos com onfalocele diagnosticados no final do primeiro e início do segundo trimestre da gestação, e todos os 8 casos apresentaram cariótipo anormal. $\mathrm{Na}$ presente série, com exceção de um caso diagnosticado neste Serviço com 13 semanas de gestação e translucência nucal normal, todos os outros foram encaminhados no segundo ou terceiro trimestres, sem referência à medida da translucência nucal.

Heydanus et al. ${ }^{11}$ não observaram alteração no volume de líquido amniótico com a onfalocele. No presente estudo, encontramos uma maior ocorrência de poliidrâmnio $(29 \%, 15 / 51)$ em relação ao oligoidrâmnio $(12 \%, 6 / 51)$.

Séries com diagnóstico pós-natal referem que a sindrome de Beckwith-Wiedemann apresenta onfalocele associada em $10-15 \%$ dos ca$\operatorname{sos}^{17}$. Observamos um caso desta síndrome com suspeita diagnóstica pré-natal sendo confirmada no pós-natal. O feto apresentava onfalocele, macroglossia e visceromegalias, com peso no nascimento de 4.950 gramas.

Sermer et al. ${ }^{14}$, observaram prematuridade (<37 semanas) em metade dos casos e incidên- cia de $35 \%$ de recém-nascidos pequenos para a idade gestacional. No presente estudo a prematuridade ocorreu com maior freqüencia no grupo com outras malformações associadas. No grupo com onfalocele isolada, $44 \%$ dos partos ocorreram antes de 37 semanas. Entretanto, a mediana da idade gestacional no parto foi de 37 semanas. A prematuridade não foi fator importante no prognóstico pós-cirúrgico, uma vez que os três casos que morreram apresentavam idade gestacional de 36 a 38 semanas.

Apesar de não ter sido demonstrada diferença significante entre os pesos no nascimento dos que sobreviveram comparados com os que morreram pós-correção cirúrgica, dois dos 3 casos que morreram apresentavam peso $\leq 2.000$ gramas. A ausência de significância pode ser justificada pelo número pequeno da amostra. Dunn e Fonkalsrud ${ }^{15}$ referem que $62 \%$ dos fetos que sobreviveram à correção cirúrgica apresentavam peso maior do que o percentil 50 no último exame ultra-sonográfico.

Kirk e Wah ${ }^{18}$ descreveram que o parto cesáreo trazia poucos benefícios em relação ao parto vaginal. A injúria ou a rotura do saco herniário e do cordão umbilical são pouco freqüentes, e não foram observadas nesta série. $\mathrm{O}$ argumento maior em favor da cesárea eletiva é o parto programado em ambiente asséptico e com preparo de todas as condições necessárias para o recém-nascido. Já Cameron et al. ${ }^{19}$ referem que a opção pela via de parto depende do tamanho e do conteúdo herniado: onfaloceles grandes teriam indicação de cesárea. Lurie et al. ${ }^{8}$ não observaram vantagem da cesárea em relação ao parto vaginal, mas recomendam que para os casos de onfaloceles grandes $(>5 \mathrm{~cm})$ seja realizada a cesárea. A carência de estudos randomizados, comparando estas duas vias de parto, justifica a polêmica e a divergência observadas na literatura. A conduta adotada no nosso serviço é de cesárea após a 38a semana, para os casos que sugerem bom prognóstico, e parto vaginal para os de mau prognóstico ou letais.

Observamos que o principal fator prognóstico nas onfaloceles com diagnóstico pré-natal são as anomalias cromossômicas e estruturais associadas, uma vez que se associam com prematuridade e baixo peso.

A conduta obstétrica pré-natal na presença de onfalocele deve incluir a investigação do cariótipo fetal e avaliação ultra-sonográfica detalhada para detecção de outras malformações associadas, incluindo-se ainda a realização de uma ecocardiografia fetal. A importância do diagnóstico pré-natal por meio da ultra-sonografia reside no diagnóstico correto da malformação e 
na identificação dos fetos que terão bom prognóstico. Esses aspectos contribuem para o aconselhamento dos pais e permitem formular um plano racional da conduta obstétrica e perinatal.

\section{SUMMARY}

Purpose: to evaluate the prognosis of fetal omphalocele after prenatal diagnosis.

Methods: fifty-one cases with prenatal diagnosis of fetal omphalocele were divided into three groups: Group 1, isolated omphalocele; Group 2, omphalocele associated with structural abnormalities and normal karyotype; Group 3, omphalocele with abnormal karyotype. The data were analyzed for overall survival rate and postsurgery survival, considering associated malformations, gestational age at delivery, birth weight and size of omphalocele.

Results: Group 1 corresponded to 21\% $(n=11)$, Group 2, $55 \%(n=28)$ and Group 3,24\% $(n=12)$. All of Group 3 died, and trisomy 18 was the most frequent chromosomal abnormality. The survival rate was $80 \%$ for Group 1 and $25 \%$ for Group 2. Sixteen cases underwent surgery (10 isolated and 6 associated), $81 \%$ survived (8 isolated and 5 associated). The median birth weight was 3,140 $\mathrm{g}$ and 2,000 $\mathrm{g}$ for survivals and non-survivals after surgery, respectively $(p=0.148)$, and the corresponding gestational age at delivery was 37 and 36 weeks $(p=0.836)$. The ratio of omphalocele/abdominal circumference decreased with gestation, 0.88 between 25-29 weeks and 0.65 between 3035 weeks $(p=0.043)$. The size of omphalocele was not significantly different between the 3 groups $(p=0.988)$, and it was not associated to postsurgery prognosis ( $p=$ $0.553)$.

Conclusion: the overall and postsurgery survival rates were 25 and 81\%, respectively. Associated malformations were the main prognostic factor in prenatally diagnosed omphaloceles, since they are associated with prematurity and low birth weight.

KEY WORDS: Fetal malformation. Omphalocele. Prenatal diagnosis. Ultrasonography.

\section{Referências}

1. Sadler TW. Digestive system; In: Langman's medical embryology. $6^{\text {th }}$ ed. Baltimore: Williams \& Wilkins; 1990. p.237-60.

2. Calzolari E, Bianchi F, Dolk H, Milan M. Omphalocele and gastroschisis in Europe: a survey of 3 million births 1980-1990. Am J Med Genet 1995; 58:18794.
3. Moore KL. The digestive system; In: The developing human. 3rd ed. Philadelphia: W.B. Saunders; 1982. p.227-55.

4. St-Vil D, Shaw KS, Lallier M, et al. Chromosomal anomalies in newborns with omphalocele. J Pediatr Surg 1996; 31:831-4.

5. Nakayama D K, Harrison M R, Gross B H, et al. Management of the fetus with an abdominal wall defect. J Pediatr Surg 1984; 19:408-13.

6. Benacerraf BR, Saltzman DH, Estroff JA, Frigoletto FD Jr. Abnormal karyotype of fetuses with omphalocele: prediction based on omphalocele contents. Obstet Gynecol 1990; 75:317-9.

7. Snijders RJ, Brizot ML, Farias M, Nicolaides KH. Fetal exomphalos at 11 to 14 weeks of gestation. J Ultrasound Med 1995; 14:569-74.

8. Lurie S, Sherman D, Bukovsky I. Omphalocele delivery enigma: the best mode of delivery still remains dubious. Eur J Obstet Gynecol Reprod Biol 1999; 82:19-22.

9. Mayer T, Black R, Matlak ME, Johnson DG. Gastroschisis and omphalocele: an eight-year review. Ann Surg 1980; 192:783-7.

10.Baird PA, MacDonald EC. An epidemiologic study of congenital malformation of the abdominal wall in more than half a million consecutive live births. Am J Hum Genet 1981; 33:470-8.

11. Heydanus R, Raats MAM, Tibboel D, Los FJ, Wladimiroff WJ. Prenatal diagnosis of fetal abdominal wall defects: a retrospective analysis of 44 cases. Prenat Diagn 1996; 16:411-7.

12.Van Zalen-Sprock RM, Vugt JMG, Van Geijn HP. First-trimester sonography of physiological midgut herniation and early diagnosis of omphalocele. Prenat Diagn 1997; 17:511-8.

13.Hughes MD, Nyberg DA, Mack LA, Pretorius DH. Fetal omphalocele: prenatal US detection of concurrent anomalies and other predictors of outcome. Radiology 1989; 173:371-6.

14.Sermer M, Benzie RJ, Pitson L, Carr M, Skidmore M. Prenatal diagnosis and management of congenital defects of the anterior abdominal wall. Am J Obstet Gynecol 1987; 156:308-12.

15.Dunn JC, Fonkalsrud EW. Improved survival of infants with omphalocele. Am J Surg 1997; 173:284-7.

16. Kamata S, Ishikawa S, Usui N, et al. Prenatal diagnosis of abdominal wall defects and their prognosis. J Pediatr Surg 1996; 31:267-71.

17. Mabogunje AO, Mahour GH. Omphalocele and gastroschisis. Trends in survival across two decades. Am J Surg 1984; 148:679-86.

18.Kirk EP, Wah RM. Obstetric management of the fetus with omphalocele or gastroschisis: A review and report of one hundred twelve cases. Am J Obstet Gynecol 1983 ;146:512-8.

19. Cameron GM, McQuown DS, Modanlou HD, Zemlyn S, Pillsbury SG Jr. Intrauterine diagnosis of an omphalocele by diagnostic ultrasonography. Am J Obstet Gynecol 1978; 131:821-2. 